\title{
Dissecting Contractile Ring Assembly Using a Multimode Microtechnique
}

\author{
K-F. Tseng, W. Chen, and D. Zhang \\ Department of Zoology and CGRB, Oregon State University, Corvallis, OR 97331
}

Micromanipulation is a simple and direct technique for studying cytoskeletal dynamics [1]. The technique, however, had been limited to bright field microscopy due to the invisibility of the microneedle by fluorescence microscopy. Thus, fluorescent analog cytochemistry [2], a widely used approach for labeling cytoskeletal elements, could not be combined with micromanipulation to study dynamics of the cytoskeleton. To overcome this limitation, we have developed light-emitting microneedles for use with a multimode microsurgery and imaging system (Fig. 1A). The system combines classic micromanipulation and microinjection with modern laser microbeam surgery on a microscope equipped for both digital-enhanced polarization and spinning disc confocal microscopy. This technique permits direct micromanipulation of both unlabeled (Fig. 2) and fluorescently tagged cytoskeletal components in vitro (Fig. 1B) and in living cells (Fig. 1C-E). Thus, labeled microtubules and actin filaments can be micromanipulated while observing their dynamics with respect to cellular events.

We are using this multimode microtechnique to address basic questions regarding the mechanics and controls of cell division. In particular, by mechanically dissecting, redistributing, and/or removing fluorescently-labeled spindle microtubules in grasshopper spermatocytes, we aim to dissect confounding mechanisms in contractile ring assembly.

It is known that contractile ring assembly involves interactions between spindle microtubules and the cell cortex. The nature of such interactions, however, remains to be determined. Here, we have directly probed the interactions between peripheral spindle microtubules and cortical actin filaments by micromanipulation in living grasshopper spermatocytes. Microtubule-actin interactions were demonstrated by tugging on the spindle or asters with a micromanipulation needle while rapidly imaging the interactions using a spinning disc confocal microscope equipped with an EM-CCD digital camera. When microtubules were dragged with a microneedle, the actin filaments at the cortex were concurrently pulled inward, causing the cell membrane to invaginate. The membrane relaxed once the needle was released. These interactions were first apparent during late anaphase and continued throughout assembly of the contractile ring. We also tested the interactions in the presence of cytochalasin D, which disassembled actin filaments into small aggregates that were mobile and readily visible. These cortical actin aggregates could ride on the tips of dynamic microtubules that were waving within and/or elongating towards the cortex (Fig. 2). In light of our recent findings in spermatocytes of silkworms [3] and grasshoppers [4], these results demonstrate a mechanism by which peripheral spindle microtubules could mechanically sweep cortical actin filaments into the incipient contractile ring.

\section{References}

[1] D. Zhang, and R.B. Nicklas Methods Cell Biol. 61 (1999) 209.

[2] Y-L. Wang Methods Cell Biol. 25 (1982) 1.

[3] W. Chen, M. Foss, K-F. Tseng, and D. Zhang PLoS Biology. (2008) 6(9): e209

[4] G.B. Alsop, W. Chen, M. Foss, K-F. Tseng, and D. Zhang PLoS One. (2009) 4(3): e4892

\footnotetext{
$\dagger$ Acknowledgment: Supported by National Science Foundation and American Heart Association.
} 


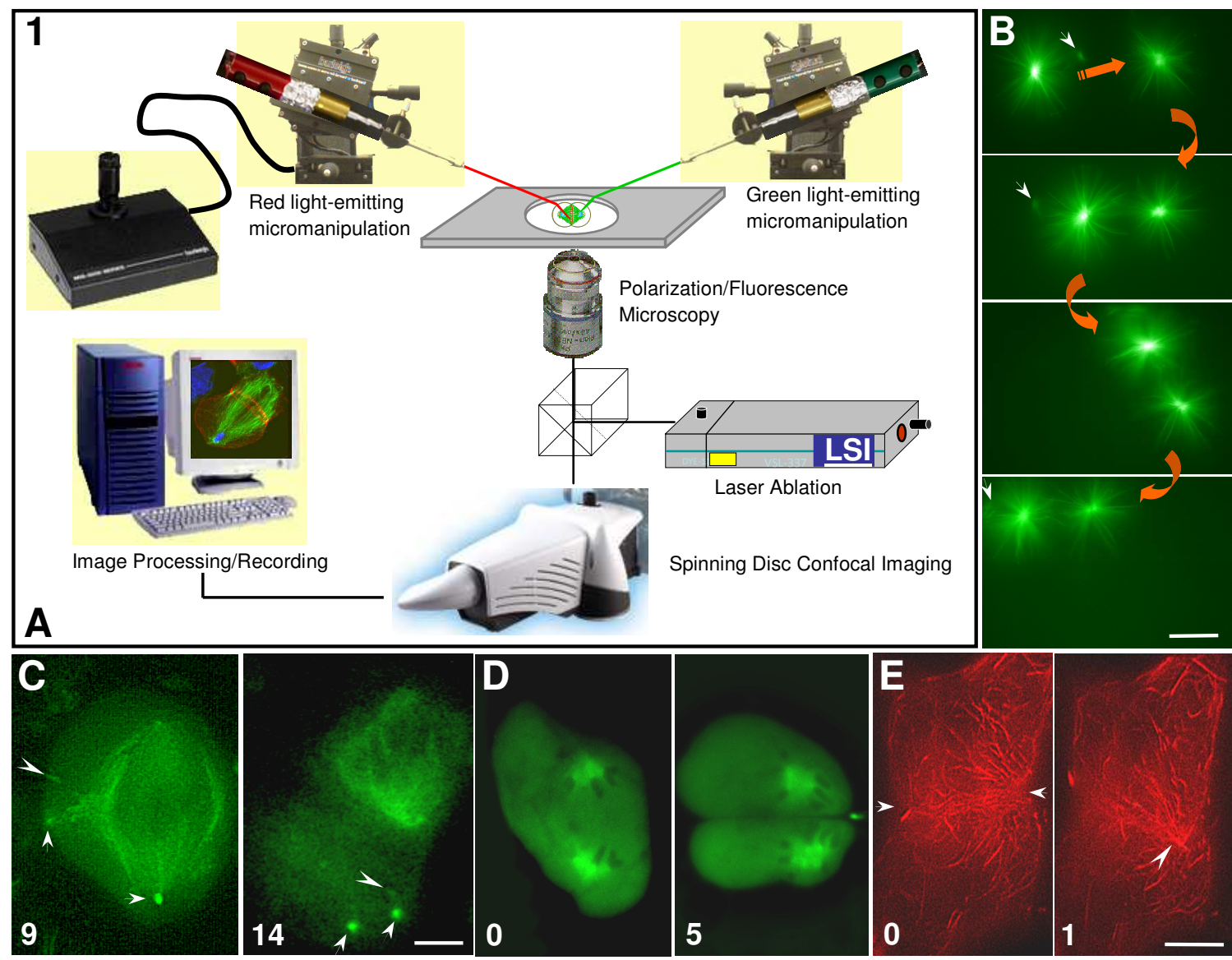

FIG. 1. A. Multimode microsurgery and imaging setup. B. Tugging asters in vitro using a green lightemitting microneedle (white arrows; red arrows depict the directions of micromanipulation). C. Detaching and secluding centrosomes (arrows) in metaphase using a green light-emitting microneedle (arrowhead). D. Cutting a cell between segregating chromosomes in anaphase using a green lightemitting microneedle. E. Dislocating actin filaments of the contractile ring (arrows) using a red lightemitting microneedle (arrowhead) in cytokinesis. Time in min., Bars, $10 \mu \mathrm{m}$.

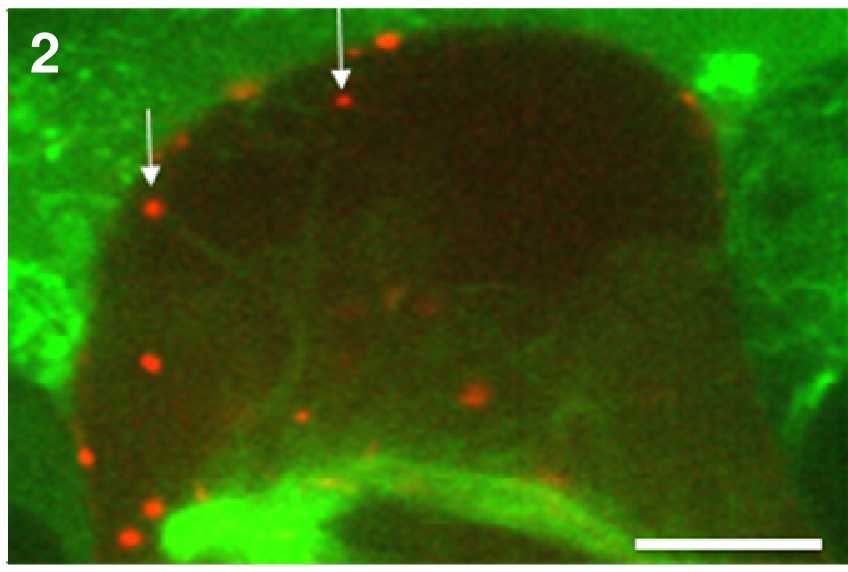

FIG. 2. Cortical actin aggregates (arrows) can ride on the tips of dynamic microtubules that are waving within and/or elongating towards the cell cortex. Bar, $10 \mu \mathrm{m}$. 